PROCEEDINGS OF THE

AMERICAN MATHEMATICAL SOCIETY

Volume 135, Number 4, April 2007, Pages 1017-1027

S 0002-9939(06)08773-9

Article electronically published on September 18, 2006

\title{
GLOBAL EXISTENCE OF SOLUTIONS TO A HYPERBOLIC-PARABOLIC SYSTEM
}

\author{
MEI ZHANG AND CHANGJIANG ZHU
}

(Communicated by Walter Craig)

\begin{abstract}
In this paper, we investigate the global existence of solutions to a hyperbolic-parabolic model of chemotaxis arising in the theory of reinforced random walks. To get $L^{2}$-estimates of solutions, we construct a nonnegative convex entropy of the corresponding hyperbolic system. The higher energy estimates are obtained by the energy method and a priori assumptions.
\end{abstract}

\section{INTRODUCTION AND MAIN RESULT}

In this paper, we consider the following system:

$$
\left\{\begin{array}{l}
u_{t}-v_{x}=0 \\
v_{t}-(u v)_{x}=v_{x x}
\end{array}\right.
$$

with boundary conditions

$$
u(0, t)=u(1, t)=0, \quad t \geq 0,
$$

and initial data

$$
u(x, 0)=u_{0}(x), \quad v(x, 0)=v_{0}(x)>0, \quad x \in[0,1] .
$$

Here the compatibility conditions on $u_{0}, v_{0}$ assume that $\left(u_{0}(0), v_{0}(0)\right)=(0,0)$, which will be used in Section 3.

Motivated by biological considerations and numerical computations carried out by Othmer and Stevens in [6] and Levine and Sleeman in [3], the system (1.1) comes from:

$$
\left\{\begin{array}{l}
\frac{\partial p}{\partial t}=D \frac{\partial}{\partial x}\left(p \frac{\partial}{\partial x}\left(\ln \frac{p}{\Phi(w)}\right)\right), \quad x \in(0, l), \quad t>0 \\
\frac{\partial w}{\partial t}=R(p, w)
\end{array}\right.
$$

where $p(x, t)$ is the particle density of a particular species, $w(x, t)$ is the concentration of the "active agent", and $D$ and $B$ are positive constants.

Received by the editors October 19, 2005.

2000 Mathematics Subject Classification. Primary 35K20, 35K55, 35L50.

Key words and phrases. Hyperbolic-parabolic system, a priori estimates, entropy-entropy flux, global existence.

(C)2006 American Mathematical Society Reverts to public domain 28 years from publication 
In fact, as in [7], let

$$
\Phi(w)=w^{-\alpha}, \quad R(p, w)=\lambda p w-\mu w,
$$

where $\alpha$ and $\lambda, \mu$ are positive constants.

Then the system (1.4) is transformed into the following form:

$$
\left\{\begin{array}{l}
p_{t}=D p_{x x}+D \alpha\left(p \frac{w_{x}}{w}\right)_{x} \\
w_{t}=\lambda p w-\mu w .
\end{array}\right.
$$

Furthermore, set

$$
q=(\ln w)_{x}=\frac{w_{x}}{w} .
$$

Then the system (1.6) can be rewritten as:

$$
\left\{\begin{array}{l}
p_{t}=D p_{x x}+D \alpha(p q)_{x} \\
q_{t}=\lambda p_{x}
\end{array}\right.
$$

Let

$$
\tau=A t, \quad \xi=l x, \quad p=B v, \quad q=c_{1} u,
$$

where $A, l$ and $c_{1}$ are positive constants to be determined below.

Then the system (1.8) becomes

$$
\left\{\begin{array}{l}
u_{\tau}=\frac{\lambda l B}{A c_{1}} v_{\xi} \\
v_{\tau}=\frac{D l^{2}}{A} v_{\xi \xi}+\frac{D \alpha l c_{1}}{A}(u v)_{\xi} .
\end{array}\right.
$$

Choosing

$$
\left\{\begin{array}{l}
\frac{\lambda l B}{A c_{1}}=1 \\
\frac{D l^{2}}{A}=1 \\
\frac{D \alpha l c_{1}}{A}=1
\end{array}\right.
$$

i.e.,

$$
A=B \alpha \lambda>0, \quad l=\sqrt{\frac{B \alpha \lambda}{D}}>0, \quad c_{1}=\sqrt{\frac{B \lambda}{\alpha D}}>0,
$$

then it is easy to verify that $u, v$ satisfy

$$
\left\{\begin{array}{l}
u_{\tau}=v_{\xi} \\
v_{\tau}=v_{\xi \xi}+(u v)_{\xi} .
\end{array}\right.
$$

If we replace $(\xi, \tau)$ by $(x, t)$, then $(1.12)$ can be rewritten as (1.1).

The system (1.4) describes the model of chemotaxis in biology. Othmer and Stevens [6] have developed a number of mathematical models of chemotaxis to illustrate aggregation leading (numerically) to nonconstant steady-states, blow-up resulting in the formation of singularities and collapse or the formation of a spatially uniform steady state. The models developed in [6] have been studied in depth by Levine and Sleeman [3. They gave some heuristic understanding of some of these phenomena and investigated the properties of solutions of a system of chemotaxis 
equations arising in the theory of reinforced random walks. Y. Yang, H. Chen and W.A. Liu [9] studied the global existence and blow-up in a finite time of solutions for the case considered in [3], respectively, and found that even at the same growth rate the behavior of the biological systems can be very different just because they started their action under different conditions. For the other results on the initial boundary value problem of (1.4) refer to [1, 4].

In this paper, we will study the global existence of solutions to the initial boundary value problem $(1.1)-(1.3)$ in $L^{\infty}\left([0, \infty), H^{2}([0,1])\right)$. To get $L^{2}$-estimates of solutions, we construct a nonnegative convex entropy of the corresponding hyperbolic system. The higher energy estimates are obtained by the energy method and a priori assumptions.

The corresponding hyperbolic system of (1.1) is

$$
\left\{\begin{array}{l}
u_{t}-v_{x}=0 \\
v_{t}-(u v)_{x}=0
\end{array}\right.
$$

The eigenvalues of (1.13) are:

$$
\lambda_{1}=-\frac{1}{2} u-\frac{1}{2} \sqrt{u^{2}+4 v}, \quad \lambda_{2}=-\frac{1}{2} u+\frac{1}{2} \sqrt{u^{2}+4 v} .
$$

Therefore, the system (1.13) is strictly hyperbolic when $v>0$.

Remark 1.1. By the boundary conditions $(1.2)$ and $(1.1)_{1}$, we have

$$
u_{t}(0, t)=u_{t}(1, t)=v_{x}(0, t)=v_{x}(1, t)=0 .
$$

Notation. Throughout this paper, we denote positive constants by $C$. Moreover, the character " $C$ " may differ in different places. $L^{p}=L^{p}([0,1])(1 \leq p \leq \infty)$ denotes the usual Lebesgue space with the norm

$$
\begin{gathered}
\|f\|_{L^{p}}=\left(\int_{0}^{1}|f(x)|^{p} d x\right)^{\frac{1}{p}}, 1 \leq p<\infty \\
|f|_{\infty}=\sup _{[0,1]}|f(x)| .
\end{gathered}
$$

$H^{l}([0,1])(l \geq 0)$ denotes the usual $l$ th-order Sobolev space with the norm

$$
\|f\|_{l}=\left(\sum_{j=0}^{l}\left\|\partial_{x}^{j} f\right\|^{2}\right)^{\frac{1}{2}},
$$

where $\|\cdot\|=\|\cdot\|_{0}=\|\cdot\|_{L^{2}}$. For simplicity, $\|f(\cdot, t)\|_{L^{p}}$ and $\|f(\cdot, t)\|_{l}$ are denoted by $\|f(t)\|_{L^{p}}$ and $\|f(t)\|_{l}$ respectively.

We will prove the following global existence theorem.

Theorem 1.2. Let $u_{0}, v_{0}-1 \in H^{2}([0,1])$ and let $\left\|u_{0}\right\|_{2}^{2}+\left\|v_{0}-1\right\|_{2}^{2}$ be sufficiently small. Then there exists a unique global solution $(u(x, t), v(x, t))$ of (1.1)-(1.3) satisfying

(i) $\quad u, v \in L^{\infty}\left([0, \infty), H^{2}([0,1])\right), \quad v_{x} \in L^{2}\left([0, \infty), H^{2}([0,1])\right)$;

$$
\text { (ii) } \quad\|u(t)\|_{2}^{2}+\|v(t)-1\|_{2}^{2}+\int_{0}^{t}\left\|v_{x}(\tau)\right\|_{2}^{2} d \tau \leq C\left(\left\|u_{0}\right\|_{2}^{2}+\left\|v_{0}-1\right\|_{2}^{2}\right) \text {. }
$$




\section{2. $L^{2}$-ENERGY ESTIMATES}

In this section, we give $L^{2}$-energy estimates of the initial boundary value problem (1.1), (1.2) and (1.3) by a nonnegative convex entropy of the system of hyperbolic conservation laws (1.13). To do this, we first give the following relation on the entropy-entropy flux pair $(\eta(u, v), q(u, v))$ of (1.13) (see [8]):

$$
\left\{\begin{array}{l}
q_{u}=-v \eta_{v} \\
q_{v}=-\eta_{u}-u \eta_{v} .
\end{array}\right.
$$

Eliminating $q$ from (2.1), we have

$$
\eta_{u u}+u \eta_{u v}-v \eta_{v v}=0 .
$$

Next, we seek the entropy of (1.13) with the following form:

$$
\eta(u, v)=\frac{1}{2} u^{2}+a(v)
$$

where $a(v)$ is a nonnegative convex function.

Substituting (2.3) into (2.2), we have

$$
1-v a^{\prime \prime}(v)=0,
$$

which implies

$$
a(v)=v \ln v-v+k_{1} v+k_{2},
$$

where $k_{1}, k_{2}$ are arbitrary constants.

It is easy to get the flux corresponding to the entropy $\eta(u, v)$ defined by $(2.3)$ and (2.4), namely

$$
q(u, v)=-u v \ln v-k_{1} u v+k_{3},
$$

where $k_{3}$ is an arbitrary constant.

In particular, if we take $k_{1}=k_{3}=0, k_{2}=1$, we will get an entropy-entropy flux pair of (1.13):

$$
\left\{\begin{array}{l}
\eta(u, v)=\frac{1}{2} u^{2}+v \ln v-v+1 \\
q(u, v)=-u v \ln v
\end{array}\right.
$$

In the next analysis, we devote ourselves to the estimates of the solution $(u(x, t)$, $v(x, t))$ of (1.1), (1.2) and (1.3) under the a priori assumptions:

$$
|u| \leq \varepsilon, \quad|v-1| \leq \frac{1}{2}, \quad\left|u_{x}\right| \leq \varepsilon, \quad\left|v_{x}\right| \leq \varepsilon,
$$

where $0<\varepsilon<<1$.

Lemma 2.1. The entropy $\eta(u, v)$ defined by $(2.6)_{1}$ satisfies for $|v-1| \leq \frac{1}{2}$,

$$
\frac{1}{2} u^{2}+\frac{1}{3}(v-1)^{2} \leq \eta(u, v) \leq \frac{1}{2} u^{2}+(v-1)^{2} .
$$

Proof. Let

$$
a_{0}(v)=v \ln v-v+1 .
$$

Then

$$
a_{0}(1)=a_{0}^{\prime}(1)=0, \quad \frac{2}{3} \leq a_{0}^{\prime \prime}(v)=\frac{1}{v} \leq 2,
$$


which implies

$$
\frac{1}{3}(v-1)^{2} \leq a_{0}(v)=\frac{1}{2} a_{0}^{\prime \prime}(\xi)(v-1)^{2} \leq(v-1)^{2},
$$

where $\xi$ is between 1 and $v$.

From $(2.6)_{1},(2.9)$ and $(2.10),(2.8)$ follows.

This proves Lemma 2.1 .

Lemma 2.2 ( $L^{2}$-energy estimates). Under the assumptions of Theorem 1.2 and the a priori assumptions (2.7), we have

$$
\int_{0}^{1}\left(\frac{1}{2} u^{2}+\frac{1}{3}(v-1)^{2}\right) d x+\frac{2}{3} \int_{0}^{t} \int_{0}^{1} v_{x}^{2} d x d \tau \leq \int_{0}^{1}\left(\frac{1}{2} u_{0}^{2}(x)+\left(v_{0}(x)-1\right)^{2}\right) d x .
$$

Proof. Multiplying (1.1) by $\nabla \eta$ and integrating it, we have by the boundary conditions (1.2) and (1.15),

$$
\int_{0}^{1} \eta(x, t) d x+\int_{0}^{t} \int_{0}^{1} \frac{v_{x}^{2}}{v} d x d s=\int_{0}^{1} \eta(x, 0) d x,
$$

$$
\int_{0}^{1}\left(\frac{1}{2} u^{2}+v \ln v-v+1\right) d x+\int_{0}^{t} \int_{0}^{1} \frac{v_{x}^{2}}{v} d x d s=\int_{0}^{1}\left(\frac{1}{2} u_{0}^{2}+v_{0} \ln v_{0}-v_{0}+1\right) d x .
$$

This proves Lemma 2.2 by Lemma 2.1 and the a priori assumptions (2.7).

\section{Higher EnERgy estimates}

In this section, we will establish higher energy estimates.

Lemma 3.1. Under the assumptions of Theorem 1.2 and the a priori assumptions (2.7), we have

$$
\int_{0}^{1}\left(u_{x}^{2}+\frac{2}{3} v_{x}^{2}\right) d x+\frac{4}{3} \int_{0}^{t} \int_{0}^{1} v_{x x}^{2} d x d s \leq C\left(\left\|u_{0}\right\|_{1}^{2}+\left\|v_{0}-1\right\|_{1}^{2}\right),
$$

where $C$ is a positive constant.

Proof. Differentiating (1.1) with respect to $x$, we have

$$
\left\{\begin{array}{l}
u_{x t}-v_{x x}=0 \\
v_{x t}-(u v)_{x x}=v_{x x x} .
\end{array}\right.
$$

For any smooth function $g_{1}(v)$, take $(3.2)_{1} \times u_{x}+(3.2)_{2} \times g_{1}(v) v_{x}$, and integrate it to get

$$
\begin{aligned}
& \frac{1}{2} \frac{d}{d t} \int_{0}^{1} u_{x}^{2} d x+\frac{1}{2} \frac{d}{d t} \int_{0}^{1} g_{1}(v) v_{x}^{2} d x \\
= & \int_{0}^{1} u_{x} v_{x x} d x+\frac{1}{2} \int_{0}^{1} g_{1}^{\prime}(v) v_{x}^{2} v_{t} d x+\int_{0}^{1} g_{1}(v) u v_{x} v_{x x} d x \\
& +2 \int_{0}^{1} g_{1}(v) u_{x} v_{x}^{2} d x+\int_{0}^{1} g_{1}(v) u_{x x} v v_{x} d x+\int_{0}^{1} g_{1}(v) v_{x} v_{x x x} d x .
\end{aligned}
$$


Next, we estimate the terms in the right side of (3.3) as follows:

$$
\begin{aligned}
\frac{1}{2} \int_{0}^{1} g_{1}^{\prime}(v) v_{x}^{2} v_{t} d x= & \frac{1}{2} \int_{0}^{1} g_{1}^{\prime}(v) v_{x}^{2}(u v)_{x} d x+\frac{1}{2} \int_{0}^{1} g_{1}^{\prime}(v) v_{x}^{2} v_{x x} d x \\
= & -\frac{1}{6} \int_{0}^{1} g_{1}^{\prime \prime}(v) v_{x}^{4} d x+\left.\frac{1}{6}\left(g_{1}^{\prime}(v) v_{x}^{3}\right)\right|_{0} ^{1} \\
& +\frac{1}{2} \int_{0}^{1} g_{1}^{\prime}(v) u v_{x}^{3} d x+\frac{1}{2} \int_{0}^{1} g_{1}^{\prime}(v) u_{x} v v_{x}^{2} d x
\end{aligned}
$$

$$
\begin{aligned}
& \int_{0}^{1} g_{1}(v) u v_{x} v_{x x} d x=-\frac{1}{2} \int_{0}^{1}\left(g_{1}(v) u\right)_{x} v_{x}^{2} d x+\left.\frac{1}{2}\left(g_{1}(v) u v_{x}^{2}\right)\right|_{0} ^{1} \\
&=-\frac{1}{2} \int_{0}^{1} g_{1}(v) u_{x} v_{x}^{2} d x-\frac{1}{2} \int_{0}^{1} g_{1}^{\prime}(v) u v_{x}^{3} d x+\left.\frac{1}{2}\left(g_{1}(v) u v_{x}^{2}\right)\right|_{0} ^{1}, \\
& \int_{0}^{1} g_{1}(v) v v_{x} u_{x x} d x=-\int_{0}^{1}\left(g_{1}(v) v v_{x}\right)_{x} u_{x} d x+\left.\left(g_{1}(v) v v_{x} u_{x}\right)\right|_{0} ^{1} \\
&=-\int_{0}^{1} g_{1}^{\prime}(v) v v_{x}^{2} u_{x} d x-\int_{0}^{1} g_{1}(v) v_{x}^{2} u_{x} d x \\
&-\int_{0}^{1} g_{1}(v) v v_{x x} u_{x} d x+\left.\left(g_{1}(v) v v_{x} u_{x}\right)\right|_{0} ^{1},
\end{aligned}
$$

and

$$
\begin{aligned}
\int_{0}^{1} g_{1}(v) v_{x} v_{x x x} d x= & -\int_{0}^{1}\left(g_{1}(v) v_{x}\right)_{x} v_{x x} d x+\left.\left(g_{1}(v) v_{x} v_{x x}\right)\right|_{0} ^{1} \\
= & \frac{1}{3} \int_{0}^{1} g_{1}^{\prime \prime}(v) v_{x}^{4} d x-\int_{0}^{1} g_{1}(v) v_{x x}^{2} d x \\
& -\left.\frac{1}{3}\left(g_{1}^{\prime}(v) v_{x}^{3}\right)\right|_{0} ^{1}+\left.\left(g_{1}(v) v_{x} v_{x x}\right)\right|_{0} ^{1} .
\end{aligned}
$$

Substituting (3.4)-(3.7) into (3.3) and using the boundary conditions (1.2) and (1.15), we obtain

$$
\begin{aligned}
& \frac{1}{2} \frac{d}{d t} \int_{0}^{1}\left(u_{x}^{2}+g_{1}(v) v_{x}^{2}\right) d x+\int_{0}^{1} g_{1}(v) v_{x x}^{2} d x \\
& \quad=\frac{1}{6} \int_{0}^{1} g_{1}^{\prime \prime}(v) v_{x}^{4} d x-\int_{0}^{1}\left(g_{1}(v) v-1\right) u_{x} v_{x x} d x-\frac{1}{2} \int_{0}^{1}\left(g_{1}^{\prime}(v) v-g_{1}(v)\right) u_{x} v_{x}^{2} d x .
\end{aligned}
$$

Choosing

$$
g_{1}(v)=\frac{1}{v}>0
$$

we have

$$
\frac{1}{2} \frac{d}{d t} \int_{0}^{1}\left(u_{x}^{2}+\frac{v_{x}^{2}}{v}\right) d x+\int_{0}^{1} \frac{v_{x x}^{2}}{v} d x=\frac{1}{3} \int_{0}^{1} \frac{v_{x}^{4}}{v^{3}} d x+\int_{0}^{1} \frac{u_{x} v_{x}^{2}}{v} d x
$$


From the a priori assumptions (2.7), we have

$$
\begin{aligned}
\frac{1}{2} \frac{d}{d t} \int_{0}^{1}\left(u_{x}^{2}+\frac{v_{x}^{2}}{v}\right) d x+\int_{0}^{1} \frac{v_{x x}^{2}}{v} d x & \leq \frac{4}{3} \varepsilon^{2} \int_{0}^{1} \frac{v_{x}^{2}}{v} d x+\varepsilon \int_{0}^{1} \frac{v_{x}^{2}}{v} d x \\
& =\left(\frac{4}{3} \varepsilon^{2}+\varepsilon\right) \int_{0}^{1} \frac{v_{x}^{2}}{v} d x
\end{aligned}
$$

Integrating (3.11) in $t$ over $[0, t]$, we can obtain

$$
\begin{aligned}
\int_{0}^{1} & \left(u_{x}^{2}+\frac{v_{x}^{2}}{v}\right) d x+2 \int_{0}^{t} \int_{0}^{1} \frac{v_{x x}^{2}}{v} d x d s \\
& \leq \int_{0}^{1}\left(u_{0 x}^{2}+\frac{v_{0 x}^{2}}{v_{0}}\right) d x+2\left(\frac{4}{3} \varepsilon^{2}+\varepsilon\right) \int_{0}^{t} \int_{0}^{1} \frac{v_{x}^{2}}{v} d x d s
\end{aligned}
$$

which implies (3.1) by (2.11) and the a priori assumptions (2.7).

The proof of Lemma 3.1 is completed.

Lemma 3.2. Under the assumptions of Theorem 1.2 and the a priori assumptions (2.7), we have

$$
\int_{0}^{1}\left(u_{x x}^{2}+\frac{2}{3} v_{x x}^{2}\right) d x+\frac{1}{3} \int_{0}^{t} \int_{0}^{1} v_{x x x}^{2} d x d s \leq C\left(\left\|u_{0}\right\|_{2}^{2}+\left\|v_{0}-1\right\|_{2}^{2}\right),
$$

where $C$ is a positive constant.

Before proving Lemma 3.2, we give the following result.

Proposition 3.3. The smooth function $v(x, t)$ obtained by Theorem 1.2 satisfies the following properties:

$$
\left|\int_{0}^{1} \frac{1}{v^{2}} v_{x x}^{3} d x\right| \leq \int_{0}^{1} \frac{1}{v} v_{x x x}^{2} d x+C \int_{0}^{1} \frac{1}{v} v_{x}^{2} d x
$$

where $C$ is a positive constant.

Proof. From the Gagliardo-Nirenberg-Moser inequality, we have

$$
\left\|v_{x x}(t)\right\|_{L^{3}}^{3} \leq C|| v_{x}(t)\left\|_{L^{6}}^{\frac{3}{2}}\right\| v_{x x x}(t) \|_{L^{2}}^{\frac{3}{2}},
$$

where $C$ is a positive constant.

(3.15) and Young's inequality show that

$$
\left\|v_{x x}(t)\right\|_{L^{3}}^{3} \leq C\left\|v_{x}(t)\right\|_{L^{6}}^{6}+\frac{2}{3}\left\|v_{x x x}(t)\right\|_{L^{2}}^{2} .
$$

Therefore, we have from the a priori assumptions (2.7),

$$
\begin{aligned}
\left|\int_{0}^{1} \frac{1}{v^{2}} v_{x x}^{3} d x\right| & \leq C|| v_{x}(t)\left\|_{L^{6}}^{6}+\frac{2}{3}\right\| v_{x x x}(t) \|_{L^{2}}^{2} \\
& \leq C\left|v_{x}\right|_{L^{\infty}}^{4} \int_{0}^{1} \frac{v_{x}^{2}}{v} d x+\int_{0}^{1} \frac{1}{v} v_{x x x}^{2} d x \\
& \leq C \int_{0}^{1} \frac{v_{x}^{2}}{v} d x+\int_{0}^{1} \frac{1}{v} v_{x x x}^{2} d x .
\end{aligned}
$$

This proves Proposition 3.3. 
Proof of Lemma 3.2. Differentiating (3.2) with respect to $x$, we have

$$
\left\{\begin{array}{l}
u_{x x t}-v_{x x x}=0 \\
v_{x x t}-(u v)_{x x x}=v_{x x x x}
\end{array}\right.
$$

For any smooth function $g_{2}(v)$, taking $(3.18)_{1} \times u_{x x}+(3.18)_{2} \times g_{2}(v) v_{x x}$, and integrating it, we have

$$
\begin{aligned}
\frac{1}{2} \frac{d}{d t} \int_{0}^{1}\left(u_{x x}^{2}+g_{2}(v) v_{x x}^{2}\right) d x= & \int_{0}^{1} u_{x x} v_{x x x} d x+\frac{1}{2} \int_{0}^{1} g_{2}^{\prime}(v) v_{t} v_{x x}^{2} d x \\
& +\int_{0}^{1} g_{2}(v) v_{x x}(u v)_{x x x} d x+\int_{0}^{1} g_{2}(v) v_{x x} v_{x x x x} d x .
\end{aligned}
$$

Next, we estimate the terms in the right side of (3.19) as follows:

$$
\begin{aligned}
\frac{1}{2} \int_{0}^{1} g_{2}^{\prime}(v) v_{t} v_{x x}^{2} d x= & \frac{1}{2} \int_{0}^{1} g_{2}^{\prime}(v)\left[(u v)_{x}+v_{x x}\right] v_{x x}^{2} d x \\
= & \frac{1}{2} \int_{0}^{1} g_{2}^{\prime}(v) u v_{x} v_{x x}^{2} d x+\frac{1}{2} \int_{0}^{1} g_{2}^{\prime}(v) u_{x} v v_{x x}^{2} d x \\
& +\frac{1}{2} \int_{0}^{1} g_{2}^{\prime}(v) v_{x x}^{3} d x,
\end{aligned}
$$

$$
\begin{aligned}
\int_{0}^{1} g_{2}(v) v_{x x}(u v)_{x x x} d x= & -\int_{0}^{1}(u v)_{x x}\left(g_{2}(v) v_{x x}\right)_{x} d x+\left.\left((u v)_{x x} g_{2}(v) v_{x x}\right)\right|_{0} ^{1} \\
= & -\int_{0}^{1} u g_{2}(v) v_{x x} v_{x x x} d x-2 \int_{0}^{1} g_{2}(v) u_{x} v_{x} v_{x x x} d x \\
& -\int_{0}^{1} v g_{2}(v) u_{x x} v_{x x x} d x-\int_{0}^{1} u g_{2}^{\prime}(v) v_{x} v_{x x}^{2} d x \\
& -2 \int_{0}^{1} g_{2}^{\prime}(v) u_{x} v_{x}^{2} v_{x x} d x+\int_{0}^{1}\left(v g_{2}^{\prime}(v) v_{x} v_{x x}\right)_{x} u_{x} d x \\
& -\left.\left(v g_{2}^{\prime}(v) v_{x} u_{x} v_{x x}\right)\right|_{0} ^{1}+\left.\left(u g_{2}(v) v_{x x}^{2}\right)\right|_{0} ^{1} \\
& +\left.2\left(g_{2}(v) u_{x} v_{x} v_{x x}\right)\right|_{0} ^{1}+\left.\left(v g_{2}(v) u_{x x} v_{x x}\right)\right|_{0} ^{1}
\end{aligned}
$$

and

$$
\begin{aligned}
\int_{0}^{1} g_{2}(v) v_{x x} v_{x x x x} d x= & -\int_{0}^{1}\left(g_{2}(v) v_{x x}\right){ }_{x} v_{x x x} d x+\left.\left(g_{2}(v) v_{x x} v_{x x x}\right)\right|_{0} ^{1} \\
= & -\int_{0}^{1} g_{2}(v) v_{x x x}^{2} d x-\int_{0}^{1} g_{2}^{\prime}(v) v_{x} v_{x x} v_{x x x} d x \\
& +\left.\left(g_{2}(v) v_{x x} v_{x x x}\right)\right|_{0} ^{1}
\end{aligned}
$$


Substituting (3.20)-(3.22) into (3.19), we obtain by the boundary conditions (1.2) and (1.15),

$$
\begin{aligned}
& \frac{1}{2} \frac{d}{d t} \int_{0}^{1}\left(u_{x x}^{2}+g_{2}(v) v_{x x}^{2}\right) d x+\int_{0}^{1} g_{2}(v) v_{x x x}^{2} d x \\
= & \int_{0}^{1} u_{x x} v_{x x x} d x-\int_{0}^{1} v g_{2}(v) u_{x x} v_{x x x} d x+\frac{1}{2} \int_{0}^{1} g_{2}^{\prime}(v) u v_{x} v_{x x}^{2} d x \\
& +\frac{1}{2} \int_{0}^{1} g_{2}^{\prime}(v) u_{x} v v_{x x}^{2} d x+\frac{1}{2} \int_{0}^{1} g_{2}^{\prime}(v) v_{x x}^{3} d x-\int_{0}^{1} u g_{2}(v) v_{x x} v_{x x x} d x \\
& -2 \int_{0}^{1} g_{2}(v) u_{x} v_{x} v_{x x x} d x-\int_{0}^{1} u g_{2}^{\prime}(v) v_{x} v_{x x}^{2} d x-2 \int_{0}^{1} g_{2}^{\prime}(v) u_{x} v_{x}^{2} v_{x x} d x \\
& +\int_{0}^{1} g_{2}^{\prime}(v) v_{x}^{2} v_{x x} u_{x} d x+\int_{0}^{1} v g_{2}^{\prime \prime}(v) v_{x}^{2} v_{x x} u_{x} d x+\int_{0}^{1} v g_{2}^{\prime}(v) v_{x x}^{2} u_{x} d x \\
& +\int_{0}^{1} v g_{2}^{\prime}(v) v_{x} v_{x x x} u_{x} d x-\int_{0}^{1} g_{2}^{\prime}(v) v_{x} v_{x x} v_{x x x} d x \\
& +\left.\left(g_{2}(v) v_{x x}\left(v u_{x x}+v_{x x x}\right)\right)\right|_{0} ^{1} .
\end{aligned}
$$

If choosing $g_{2}(v)=\frac{1}{v}$, then we get

$$
\begin{aligned}
& \frac{1}{2} \frac{d}{d t} \int_{0}^{1}\left(u_{x x}^{2}+\frac{1}{v} v_{x x}^{2}\right) d x+\int_{0}^{1} \frac{1}{v} v_{x x x}^{2} d x \\
= & \frac{1}{2} \int_{0}^{1} \frac{u}{v^{2}} v_{x} v_{x x}^{2} d x-\frac{1}{2} \int_{0}^{1} \frac{1}{v} u_{x} v_{x x}^{2} d x-\frac{1}{2} \int_{0}^{1} \frac{1}{v^{2}} v_{x x}^{3} d x \\
& -\int_{0}^{1} \frac{u}{v} v_{x x} v_{x x x} d x-3 \int_{0}^{1} \frac{1}{v} u_{x} v_{x} v_{x x x} d x+3 \int_{0}^{1} \frac{1}{v^{2}} v_{x}^{2} u_{x} v_{x x} d x \\
& -\int_{0}^{1} \frac{1}{v} u_{x} v_{x x}^{2} d x+\int_{0}^{1} \frac{1}{v^{2}} v_{x} v_{x x} v_{x x x} d x+\left.\left(\frac{v_{x x}}{v}\left(v u_{x x}+v_{x x x}\right)\right)\right|_{0} ^{1} .
\end{aligned}
$$

From $(3.2)_{2}$ and the boundary conditions (1.2) and (1.15), we have

$$
\left.v_{x x x}\right|_{0} ^{1}=-\left.\left((u v)_{x x}\right)\right|_{0} ^{1}=-\left.\left(u v_{x x}\right)\right|_{0} ^{1}-\left.2\left(u_{x} v_{x}\right)\right|_{0} ^{1}-\left.\left(u_{x x} v\right)\right|_{0} ^{1}=-\left.\left(u_{x x} v\right)\right|_{0} ^{1} .
$$

Thus

$$
\left.\left(\frac{v_{x x}}{v}\left(v u_{x x}+v_{x x x}\right)\right)\right|_{0} ^{1}=0 .
$$

By (3.24), (3.25), (3.14) and using the Cauchy-Schwarz inequality, we have

$$
\begin{aligned}
& \frac{1}{2} \frac{d}{d t} \int_{0}^{1}\left(u_{x x}^{2}+\frac{1}{v} v_{x x}^{2}\right) d x+\int_{0}^{1} \frac{1}{v} v_{x x x}^{2} d x \\
\leq & \frac{3}{4} \int_{0}^{1} \frac{1}{v} v_{x x x}^{2} d x+C \int_{0}^{1} \frac{1}{v}\left(v_{x}^{2}+v_{x x}^{2}\right) d x .
\end{aligned}
$$

Integrating (3.26) in $t$ over $[0, t]$ and using Lemmas 2.2 and 3.1, and the a priori assumptions (2.7), we get (3.13).

This proves Lemma 3.2 . 


\section{The PRoOF of TheOREM 1.2}

The global existence in Theorem 1.2 follows from a local existence theorem (see [2, 5]) and the a priori estimate (1.16) obtained by (2.11), (3.1) and (3.13).

Now, we have to show that the a priori assumptions (2.7) can be closed since, under the a priori assumptions (2.7), we have proved that (1.16) holds.

In fact, by Sobolev's embedding theorem $W^{1,1}([0,1]) \hookrightarrow L^{\infty}([0,1])$ and Hölder's inequality, we have

$$
\begin{aligned}
|v(x, t)-1| & \leq C \int_{0}^{1}|v(x, t)-1| d x+C \int_{0}^{1}\left|\frac{\partial}{\partial x}(v(x, t)-1)\right| d x \\
& \leq C\left(\int_{0}^{1}(v(x, t)-1)^{2} d x\right)^{\frac{1}{2}}+C\left(\int_{0}^{1} v_{x}^{2}(x, t) d x\right)^{\frac{1}{2}} \\
& \leq C\left(\left\|u_{0}\right\|_{1}^{2}+\left\|v_{0}-1\right\|_{1}^{2}\right)^{\frac{1}{2}}
\end{aligned}
$$

which implies

$$
|v(x, t)-1| \leq C\left(\left\|u_{0}\right\|_{2}^{2}+\left\|v_{0}-1\right\|_{2}^{2}\right)^{\frac{1}{2}}
$$

Similarly, we have

$$
|u(x, t)|, \quad\left|u_{x}(x, t)\right|, \quad\left|v_{x}(x, t)\right| \leq C\left(\left\|u_{0}\right\|_{2}^{2}+\left\|v_{0}-1\right\|_{2}^{2}\right)^{\frac{1}{2}} .
$$

By (4.1) and (4.2), it is easy to see that the a priori assumptions (2.7) hold provided $\left\|u_{0}\right\|_{2}^{2}+\left\|v_{0}-1\right\|_{2}^{2}$ is sufficiently small. Therefore, the a priori assumptions (2.7) are always true provided $\left\|u_{0}\right\|_{2}^{2}+\left\|v_{0}-1\right\|_{2}^{2}$ is sufficiently small.

\section{ACKNOWLEDGEMENT}

The research was supported by the Program for New Century Excellent Talents in University \#NCET-04-0745, the Key Project of the National Natural Science Foundation of China \#10431060. Special thanks go to the anonymous referee for his/her helpful comments on the draft version of this manuscript.

\section{REFERENCES}

[1] T. Hillen, A. Potapov, The one-dimensional chemotaxis model: global existence and asymptotic profile, Math. Methods Appl. Sci., 27(2004), 1783-1801. MR2087297(2005e:35100)

[2] S. Kato, On local and global existence theorems for a nonautonomous differential equation in a Banach space, Funkcial. Ekvac., 19(1976), 279-286. MR0440149 (55:13029)

[3] H.A. Levine, B.D. Sleeman, A system of reaction diffusion equations arising in the theory of reinforced random walks, SIAM J. Appl. Math., 57(1997), 683-730. MR1450846 (98g:35106)

[4] H.A. Levine, B.D. Sleeman, M.N. Hamilton, Mathematical modeling of the onset of capillary formation initiating angiogenesis, J. Math. Biol., 42(2001), 195-238. MR.1828815 (2003b:92003)

[5] T. Nishida, Nonlinear hyperbolic equations and related topics in fluid dynamics, Publ. Math., 1978. MR0481578 (58:1690)

[6] H.G. Othmer, A. Stevens, Aggregation, blowup, and collapse: the ABC's of taxis in reinforced random walks, SIAM J. Appl. Math., 57(1997), 1044-1081. MR.1462051 (99b:92001)

[7] B.D. Sleeman, H.A. Levine, Partial differential equations of chemotaxis and angiogenesis, Math. Methods. Appl. Sci., 24(2001), 405-426. MR.1821934 (2002c:92009) 
[8] J. Smoller, Shock Waves and Reaction-Diffusion Equations, Springer-Verlag, New YorkBerlin, 1983. MR0688146 (84d:35002)

[9] Y. Yang, H. Chen, W.A. Liu, On existence of global solutions and blow-up to a system of reaction-diffusion equations modelling chemotaxis, SIAM J. Math. Anal., 33(2001), 763-785. MR.1884721 (2003d:35159)

Laboratory of Nonlinear Analysis, Department of Mathematics, Central China Normal University, Wuhan 430079, People's Republic of China

Laboratory of Nonlinear Analysis, Department of Mathematics, Central China Normal University, Wuhan 430079, People's Republic of China

E-mail address: cjzhu@mail.ccnu.edu.cn 